\title{
Number of retrieved lymph nodes is an independent prognostic factor after total gastrectomy for patients with stage III gastric cancer: propensity score matching analysis of a multi-institution dataset
}

\author{
Shogo Hayashi ${ }^{1} \cdot$ Mitsuro Kanda $^{2}$ (D) Seiji Ito ${ }^{3} \cdot$ Yoshinari Mochizuki $^{4} \cdot$ Hitoshi Teramoto $^{5} \cdot$ Kiyoshi Ishigure $^{6}$. \\ Toshifumi Murai ${ }^{7} \cdot$ Takahiro Asada $^{8}$ · Akiharu Ishiyama ${ }^{9} \cdot$ Hidenobu Matsushita $^{1} \cdot$ Chie Tanaka $^{2}$. \\ Daisuke Kobayashi $^{2} \cdot$ Michitaka Fujiwara $^{2} \cdot$ Kenta Murotani $^{10} \cdot$ Yasuhiro Kodera $^{2}$
}

Received: 31 August 2018 / Accepted: 20 November 2018 / Published online: 27 November 2018

(c) The International Gastric Cancer Association and The Japanese Gastric Cancer Association 2018

\begin{abstract}
Background The prognostic significance of the number of retrieved lymph nodes (RLNs) in gastric cancer remains controversial. Therefore, we designed a multicenter collaborative database to investigate the correlation between the number of RLNs and prognosis of patients with advanced gastric cancer after curative resection.

Methods We retrospectively analyzed 1103 patients who underwent gastrectomy for stage II/III gastric cancer between 2010 and 2014. Lymph nodes, which were retrieved by surgeons from surgically resected specimens, were validated by pathologists. A target population and the optimal cutoff were determined using receiver operating characteristic (ROC) curve analysis. After propensity score matching of eight variables, including splenectomy and adjuvant chemotherapy, the prognostic significance of RLNs was evaluated.

Results According to ROC curve analysis, the optimum cutoff score for predicting postoperative survival was 40 . After matching, the backgrounds of patients in the RLN $<40$ and $\mathrm{RLN} \geq 40$ groups $(n=87$ each) became well-balanced. The RLN $<40$ group experienced significantly shorter relapse-free and overall survival. The prevalence of peritoneal recurrence was significantly increased in the RLN $<40$ group. RLN $<40$ was an independent prognostic factor in multivariable analysis, although pathological $\mathrm{N}$ status was not. A forest plot revealed that the RLN $<40$ group was at greater risk of recurrence in most subgroups.

Conclusions $\mathrm{RLN}<40$ was associated with an adverse prognosis of patients with stage III gastric cancer who underwent total gastrectomy.
\end{abstract}

Keywords Gastric cancer · Number of retrieved LNs · Prognosis · Total gastrectomy

Electronic supplementary material The online version of this article (https://doi.org/10.1007/s10120-018-0902-2) contains supplementary material, which is available to authorized users.

Mitsuro Kanda

m-kanda@med.nagoya-u.ac.jp

1 Department of Surgery, Tosei General Hospital, Seto, Japan

2 Department of Gastroenterological Surgery (Surgery II), Nagoya University Graduate School of Medicine, 65 Tsurumai-cho, Showa-ku, Nagoya 466-8550, Japan

3 Department of Gastroenterological Surgery, Aichi Cancer Center, Nagoya, Japan

4 Department of Surgery, Komaki Municipal Hospital, Komaki, Japan

5 Department of Surgery, Yokkaichi Municipal Hospital, Yokkaichi, Japan
6 Department of Surgery, Konan Kosei Hospital, Konan, Japan

7 Department of Surgery, Ichinomiya Municipal Hospital, Ichinomiya, Japan

8 Department of Surgery, Gifu Prefectural Tajimi Hospital, Tajimi, Japan

9 Department of Surgery, Okazaki City Hospital, Okazaki, Japan

10 Biostatistics Center, Graduate School of Medicine, Kurume University, Kurume, Japan 


\section{Introduction}

Recent advances in screening systems and endoscopic instruments have enabled the early diagnosis of gastric cancer, and minimally invasive treatment of patients with early gastric cancer achieves an excellent prognosis [1,2]. Unfortunately, many patients have advanced gastric cancer at the time of diagnosis and die as a result [3]. The best treatment of advanced gastric cancer is radical gastrectomy with systematic lymph node (LN) dissection and adjuvant chemotherapy [4, 5]. Nodal metastasis is an important determinant of recurrence and longterm survival of patients undergoing radical gastrectomy, and management of nodal disease is a key component of ensuring the best survival outcomes $[3,6]$.

Although systematic lymphadenectomy is considered a pivotal strategy for macroscopic tumor clearance in advanced gastric cancer, there are certain differences in the numbers of retrieved LNs (RLNs), even if surgery is performed as recommended by the Japanese Gastric Cancer Treatment Guideline [7-9]. The number of RLNs is influenced by factors such as the extent of lymphadenectomy and gastrectomy, intra-abdominal fat volume, innate number of nodes in each patient, and a surgeon's enthusiasm for harvesting more nodes [10, 11]. In the Japanese Classification of Gastric Carcinoma Carcinoma, 15th Edition [6] and the TNM Classification of Malignant Tumors, 8th Edition [12] (TNM), at least 16 RLNs are recommended for appropriate staging; however, information is not available that indicate prognostic risks if an unsatisfactory number of RLNs are obtained [7, 12]. Numerous studies report that the presence, position, and number of positive LNs serve as significant prognostic factors $[13,14]$. However, the number of LNs linked to prognosis is known only for certain gastrointestinal malignancies [9, 15, 16]. Moreover, it is unclear whether variables such as surgical procedure, range of lymphadenectomy, and disease stage influence the ability of the number of RLNs to predict prognosis of patients with resectable but advanced gastric cancer.

The purpose of the present study was to investigate the correlation between the number of RLNs in patients with advanced gastric cancer who underwent curative resection with their postoperative prognosis. To this end, we conducted an analysis of a multicenter collaborative database. Our findings strongly support the conclusion that using an optimum cutoff value for the number of RLNs facilitates the prediction of prognosis of such patients.

\section{Patients and methods}

\section{Patient selection}

We retrospectively collected the clinical data from nine institutions for 3484 patients who underwent gastrectomy for gastric cancer between January 2010 and December 2014. Before analyzing the data, we classified these patients according to the TNM classification [12] and the Japanese Gastric Cancer Treatment Guideline [7]. We selected 1103 patients for analysis according to the inclusion criteria as follows: no preoperative treatment, R0 gastrectomy with systematic lymphadenectomy performed according to the Japanese Gastric Cancer Treatment Guideline [7], pathological stage II-III gastric cancer according to the TNM classification [11], and sufficient data for analysis (Fig. 1a). We excluded patients with gastric stump cancer, those who underwent extended surgery (e.g. pancreaticoduodenectomy and Appleby's procedure) or gastrectomy without systemic lymphadenectomy, and those with a postoperative follow-up $<3$ months. We excluded patients with missing or insufficient data for RLNs, pathological findings, macroscopic tumor size, or preoperative serum tumor markers.

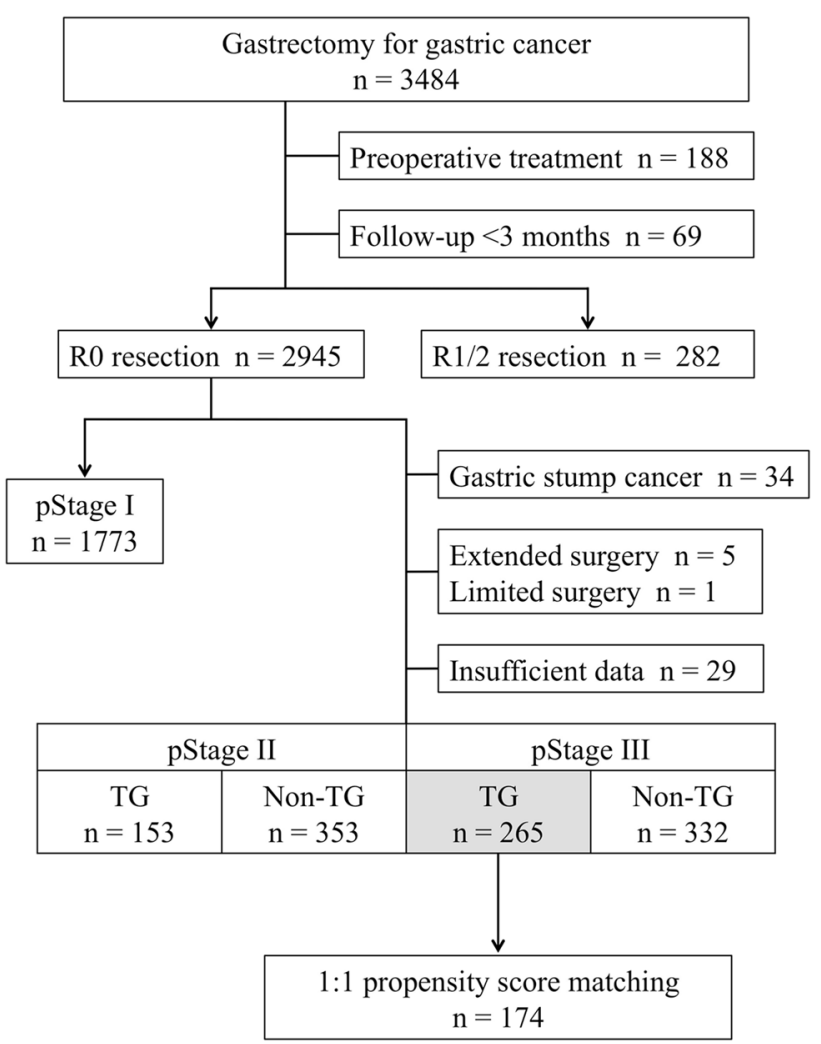

Fig. 1 Study design 
This study conformed to the ethical guidelines of the World Medical Association Declaration of Helsinki-Ethical Principles for Medical Research Involving Human Subjects. Written informed consent for surgery and use of clinical data was obtained from patients as required by the Institutional Review Board of each participating institution. We applied opt-out recruitment according to the policy of the Japanese government because we conducted clinical research using the only retrospective clinical data without intervention. The purpose, design, and objectives of the study were posted on our homepage (https://www.med.nagoya-u.ac.jp/medical_J/ ethics/rinsyoukansatsu.html) to provide an opportunity for patients to decline to contribute to our study.

\section{Patient management}

Patients underwent gastrectomy with systematic lymphadenectomy (D1+ for clinical T1N0 patients and D2 for the others) according to the Japanese Gastric Cancer Treatment Guideline [7]. The reconstruction method was selected at the surgeon's discretion. The indication for splenectomy was determined by the attending physicians according to tumor location and suspicion of nodal metastasis around the splenic hilum. Patients received postoperative follow-up that included physical examinations and the laboratory tests as follows: serum tumor markers 1 month after surgery and every 3 months thereafter, and enhanced computed tomography (chest and abdominal cavity) once every 6 months for 5 years or until recurrence, according to the Japanese Gastric Cancer Treatment Guideline [7]. Further, patients underwent enhanced computed tomography if their symptoms and blood tests indicated a recurrence. 12 months of S-1 (an oral fluoropyrimidine derivative) or 6 months of capecitabine plus oxaliplatin was recommended to all patients as postoperative adjuvant treatment unless contraindicated by the patient's condition or refusal [17]. Treatment after recurrence was determined according to the evidence available at the time of treatment and the patient's condition and consent.

\section{Quality of surgery}

To ensure the quality of gastrectomy and systemic lymphadenectomy, the institutes we selected institutions performed $>50$ gastrectomies for gastric cancer annually in accordance with the Japanese Gastric Cancer Treatment Guideline [6]. Surgeons with board certifications from the Japanese Society of Gastroenterological Surgery performed or supervised all surgeries. A central review was not performed.

\section{Retrieval of LNs}

The extent of LN dissection was based on the Japanese Gastric Cancer Treatment Guideline [7]. After removal of the stomach and surrounding tissues, surgeons retrieved as many LNs as possible from the resected specimen, and pathologists counted the RLNs and validated their characteristics [18].

\section{Correlations between the number of RLNs and prognosis}

We determined a target population and the optimal cutoff value of RLNs to predict 2-year survival after surgery using receiver operating characteristic (ROC) curve analysis, according to the type of gastrectomy (total or partial) and pathological disease stage (II or III).

\section{Propensity score matching}

We employed propensity score matching to strictly balance the significant variables used in the analyses that follow. Propensity scores were estimated using a logistic regression model according to age, sex, preoperative body mass index, splenectomy, open or laparoscopic surgery, macroscopic tumor size, estimated intraoperative blood loss, and adjuvant chemotherapy. One-to-one matching without replacement was performed using a 0.1 caliper width, and the resulting score-matched pairs were used in subsequent analyses.

\section{Statistical analysis}

The primary endpoint of this study was to evaluate the prognostic significance of the number of RLNs in patients who underwent curative resection to treat advanced gastric cancer. The secondary endpoints included the identification of a target population (type of gastrectomy and disease stage), determining an optimal cutoff value of the number of RLNs to predict prognosis, and subgroup analyses. Qualitative variables were compared between the two patient groups using the $\chi^{2}$ test, and quantitative variables were compared using the Mann-Whitney test. Survival rates were estimated using the Kaplan-Meier method. Patients who died from causes other than gastric cancer were censored in the survival analyses. The Cox proportional hazards model and multivariable analysis were used to evaluate the hazard ratio relative to each variable. Statistical analyses were performed using SAS version 9.4 and JMP version 10 (SAS Institute, Cary, NC). $P<0.05$ was considered statistically significant. 


\section{Results}

\section{Selection of a patient group with the largest prognostic association with the number of RLNs}

The characteristics of 1103 patients with stage II/III gastric cancer are shown in Supplemental Table 1. Total gastrectomy was administered to 418 patients (38\%) (mean RLNs $=37.7)$. Patients were pathologically diagnosed as stages IB $(n=60)$, IIA $(n=263)$, IIB $(n=243)$, IIIA $(n=325)$, IIIB $(n=193)$, and IIIC $(n=79)$. The median follow-up was 42.4 months.

ROC analysis of patients who underwent total gastrectomy for stage III gastric cancer revealed that the number of RLNs had the largest influence on prognosis 2 years after surgery (Supplemental Fig. 1). Accordingly, we further analyzed the stage III - total gastrectomy group $(n=265)$. The optimum discriminative cutoff for the number of RLNs was set to 40. Using this cutoff, we classified 265 patients into the RLN $<40(n=115)$ and $\mathrm{RLN} \geq 40(n=150)$ groups. Before propensity score matching, the RLN $<40$ group had a significantly greater proportion of elderly patients, higher prevalence of cardiovascular comorbidities, and splenectomies (Table 1). Further, more $\mathrm{pN} 3$ patients were included in the $\mathrm{RLN} \geq 40$ group. Propensity score matching was performed to minimize selection bias, and the clinicopathological characteristics of the two groups $(n=87$, each) became well-balanced as demonstrated by reduced standardized differences (Table 1). The median follow-up periods were 37.3 months and 46.3 months for the RLN $<40$ and RLN $\geq 40$ groups, respectively.

\section{Treatment profile of the matched cohort}

S-1 monotherapy was administered to 125 patients who underwent postoperative adjuvant chemotherapy because such chemotherapy was the standard treatment for patients with pathological Stage III during the study period (2010-2014). All participating institutes employed the same strategy. S-1 monotherapy was administered to 115 (92\%) patients. In clinical trials of postoperative adjuvant chemotherapy, 5 (4\%) patients received S-1 plus docetaxel and $5(4 \%)$ patients received $S-1$ plus cisplatin. There was no significant difference in the proportion of doublet chemotherapies between the RLN $<40$ and $\mathrm{RLN} \geq 40$ groups (8.1\% and $3.5 \%$, respectively, $P=0.1870$ ).

\section{Prognostic significance of the number of RLNs}

The RLN $\geq 40$ group experienced significantly longer relapse-free survival (hazard ratio [HR] 2.35, 95\% confidence interval [CI] 1.51-3.72, $P=0.0001$ ) (Fig. 2a). Moreover, patients in the RLN $\geq 40$ group were more likely to experience longer overall survival (HR 2.11, 95\% CI 1.24-3.71, $P=0.0057$ ) (Fig. 2b). We detected a greater tendency in the RLN $<40$ group for the increased prevalence of peritoneal dissemination, nodal recurrence, and hematogenous recurrence. Notably, the recurrence rate of peritoneal dissemination was significantly increased in the RLN $<40$ group (Fig. 2c). In multivariate analysis, the $\mathrm{RLN}<40$ group had the second-highest HR for candidate risk factors (HR 2.43; 95\% CI 1.54-3.94, $P=0.0001$ ) (Table 2). Further, we identified pathological lymph node metastasis (pN3) and administration of adjuvant chemotherapy as independent prognostic factors. Interestingly, macroscopic tumor size, pathological $\mathrm{T}$ factor, and splenectomy were not significant independent prognostic factors.

\section{Subgroup analyses}

We performed subgroup analyses to further investigate the clinical significance of RLNs. A forest plot revealed that the $\mathrm{RLN}<40$ group was at greater risk of disease recurrence in most subgroups (Fig. 3). We then focused on splenectomy, nodal status, and administration of postoperative adjuvant chemotherapy. The 3-year relapse-free survival rates with or without splenectomy were $71.8 \%$ and $61.4 \%$ for patients with RLN $\geq 40$ and $38.7 \%$ and $32.2 \%$ for those with RLN $<40$, respectively. There were no statistically significant differences in relapse-free survival within the same RLN group, independent of splenectomy (Fig. 4a). The associations of RLNs on relapse-free survival were similar for both subgroups, independent of splenectomy (Supplemental Fig. 2).

When patients were stratified into pN0-2 $(n=99)$ and pN3 $(n=75)$ subgroups, the RLN $>40$ and RLN $\geq 40$ groups experienced significantly longer relapse-free survival, with hazard ratios of 2.92 (95\% CI 1.41-6.65) in the pN0-2 subgroup and 2.46 (95\% CI 1.39-4.44) in the $\mathrm{pN} 3$ subgroup (Supplemental Fig. 3). The 3-year relapse-free survival rates were $72.3 \%$ and $54.3 \%$ for the RLN $\geq 40$ subgroup with and without adjuvant chemotherapy, respectively, and $39.2 \%$ and $26.5 \%$ for patients in the RLN $<40$ with and without adjuvant chemotherapy, respectively (Fig. 4b). Compared with the difference in survival of patients who underwent surgery alone, the extension of relapse-free survival in the RLN $\geq 40$ group was longer compared with those who underwent adjuvant chemotherapy (Supplemental Fig. 4).

\section{Discussion}

In the present study, we initially explored the setting in which the RLNs most strongly influenced prognosis. For this purpose, we employed a multicenter collaborative database 
Table 1 Demographics and pathological characteristics before and after propensity score matching

\begin{tabular}{|c|c|c|c|c|c|c|}
\hline \multirow[t]{2}{*}{ Characteristic } & \multicolumn{3}{|c|}{ Unmatched comparison } & \multicolumn{3}{|c|}{ Matched comparison } \\
\hline & RLN $<40(n=115)$ & $\mathrm{RLN} \geq 40(n=150)$ & $\begin{array}{l}\text { Standardized } \\
\text { difference }\end{array}$ & $\begin{array}{l}\mathrm{RLN}<40 \\
(n=87)\end{array}$ & $\begin{array}{l}\mathrm{RLN} \geq 40 \\
(n=87)\end{array}$ & $\begin{array}{l}\text { Standardized } \\
\text { difference }\end{array}$ \\
\hline Age (years), mean \pm SD & $71.0 \pm 11.3$ & $66.2 \pm 10.4$ & 0.448 & $68.9 \pm 11.8$ & $69.2 \pm 8.4$ & 0.024 \\
\hline Sex (male/female) & $82 / 33$ & $108 / 42$ & 0.015 & $61 / 26$ & $64 / 23$ & 0.077 \\
\hline Preoperative symptom (\%) & $55(48 \%)$ & $80(53 \%)$ & 0.110 & $45(52 \%)$ & $40(46 \%)$ & 0.115 \\
\hline $\begin{array}{l}\text { Preoperative body mass index } \\
(\text { mean } \pm \text { SD })\end{array}$ & $22.2 \pm 3.4$ & $21.5 \pm 3.1$ & 0.211 & $21.8 \pm 3.5$ & $21.8 \pm 3.4$ & 0.002 \\
\hline \multicolumn{7}{|l|}{ Cardiovascular comorbidity } \\
\hline Absent & 91 & 139 & 0.397 & 75 & 77 & 0.069 \\
\hline Present & 24 & 11 & & 12 & 10 & \\
\hline \multicolumn{7}{|l|}{ Chronic pulmonary disease } \\
\hline Absent & 108 & 141 & 0.004 & 83 & 81 & 0.099 \\
\hline Present & 7 & 9 & & 4 & 6 & \\
\hline \multicolumn{7}{|l|}{ Cerebrovascular disease } \\
\hline Absent & 107 & 137 & 0.064 & 81 & 78 & 0.123 \\
\hline Present & 8 & 13 & & 6 & 9 & \\
\hline \multicolumn{7}{|l|}{ Diabetes mellitus } \\
\hline Absent & 94 & 125 & 0.042 & 74 & 69 & 0.151 \\
\hline Present & 21 & 25 & & 13 & 18 & \\
\hline \multicolumn{7}{|l|}{ Preoperative CEA (ng/ml) } \\
\hline$\leq 5.0$ & 89 & 117 & 0.015 & 69 & 67 & 0.056 \\
\hline$>5.0$ & 26 & 33 & & 18 & 20 & \\
\hline \multicolumn{7}{|l|}{ Preoperative CA19-9 (IU/ml) } \\
\hline$\leq 37$ & 95 & 119 & 0.084 & 69 & 66 & 0.083 \\
\hline$>37$ & 20 & 31 & & 18 & 21 & \\
\hline \multicolumn{7}{|l|}{ Tumor location } \\
\hline Entire & 17 & 15 & 0.15 & 10 & 9 & 0.038 \\
\hline Upper third & 61 & 85 & & 49 & 50 & \\
\hline Middle third & 29 & 38 & & 21 & 21 & \\
\hline Lower third & 8 & 12 & & 7 & 7 & \\
\hline $\begin{array}{l}\text { Macroscopic tumor size, median } \\
\text { (range) }\end{array}$ & $60(25-180)$ & $60(20-170)$ & 0.072 & $60(25-180)$ & $60(30-160)$ & 0.002 \\
\hline \multicolumn{7}{|l|}{ Surgical approach } \\
\hline Open & 109 & 145 & 0.093 & 84 & 83 & 0.059 \\
\hline Laparoscopic & 5 & 5 & & 3 & 4 & \\
\hline Splenectomy (\%) & $50(43 \%)$ & $90(60 \%)$ & 0.335 & $46(53 \%)$ & $48(55 \%)$ & 0.046 \\
\hline \multicolumn{7}{|l|}{ Lymphadenectomy } \\
\hline $\mathrm{D} 1+$ & 32 & 17 & 0.425 & 8 & 7 & 0.327 \\
\hline D2 & 83 & 133 & & 79 & 80 & \\
\hline Operative time (min), median (range) & $283(138-538)$ & $280(150-560)$ & 0.124 & $\begin{array}{l}278(159- \\
448)\end{array}$ & $\begin{array}{l}280(155- \\
449)\end{array}$ & 0.085 \\
\hline $\begin{array}{l}\text { Estimated blood loss (ml), median } \\
\text { (range) }\end{array}$ & $400(10-2465)$ & $389(0-6362)$ & 0.054 & $\begin{array}{l}400 \\
(10-2413)\end{array}$ & $370(0-3050)$ & 0.009 \\
\hline \multicolumn{7}{|l|}{ Intraoperative transfusion } \\
\hline Absent & 101 & 136 & 0.092 & 77 & 79 & 0.076 \\
\hline Present & 14 & 14 & & 10 & 8 & \\
\hline RLNs (mean $\pm \mathrm{SD})$ & $29.4 \pm 8.4$ & $56.8 \pm 15.5$ & 2.296 & $29.4 \pm 8.7$ & $56.1 \pm 12.7$ & 2.644 \\
\hline Postoperative complication $^{\mathrm{a}}(\%)$ & $47(41 \%)$ & $58(39 \%)$ & 0.045 & $35(40 \%)$ & $36(41 \%)$ & 0.023 \\
\hline \multicolumn{7}{|l|}{ Differentiation } \\
\hline Differentiated & 45 & 54 & 0.065 & 35 & 36 & 0.023 \\
\hline
\end{tabular}


Table 1 (continued)

\begin{tabular}{|c|c|c|c|c|c|c|c|}
\hline \multirow[t]{2}{*}{ Characteristic } & \multicolumn{4}{|c|}{ Unmatched comparison } & \multicolumn{3}{|c|}{ Matched comparison } \\
\hline & $\operatorname{RLN}<40(n=115)$ & $\operatorname{RLN} \geq 40(n=150)$ & \multicolumn{2}{|c|}{$\begin{array}{l}\text { Standardized } \\
\text { difference }\end{array}$} & $\begin{array}{l}\mathrm{RLN}<40 \\
(n=87)\end{array}$ & $\begin{array}{l}\mathrm{RLN} \geq 40 \\
(n=87)\end{array}$ & $\begin{array}{l}\text { Standardized } \\
\text { difference }\end{array}$ \\
\hline Undifferentiated & 70 & 96 & & & 52 & 51 & \\
\hline \multicolumn{8}{|l|}{$\mathrm{T}$ factor } \\
\hline pT1-3 & 33 & 47 & \multicolumn{2}{|l|}{0.058} & 26 & 32 & \multirow[t]{2}{*}{0.147} \\
\hline pT4 & 82 & 103 & & & 61 & 55 & \\
\hline \multicolumn{8}{|l|}{$\mathrm{N}$ factor } \\
\hline pN0 & 2 & 1 & \multirow{4}{*}{\multicolumn{2}{|c|}{0.416}} & 2 & 1 & \multirow[t]{4}{*}{0.087} \\
\hline $\mathrm{pN} 1$ & 24 & 22 & & & 15 & 14 & \\
\hline $\mathrm{pN} 2$ & 49 & 45 & & & 33 & 34 & \\
\hline $\mathrm{pN} 3$ & 40 & 82 & & & 37 & 38 & \\
\hline \multicolumn{8}{|l|}{ Pathological stage } \\
\hline IIIA & 72 & 64 & \multirow[t]{3}{*}{0.413} & 47 & & 48 & \multirow[t]{3}{*}{0.096} \\
\hline IIIB & 29 & 53 & & 26 & & 23 & \\
\hline IIIC & 14 & 33 & & 14 & & 16 & \\
\hline \multicolumn{8}{|l|}{ Adjuvant chemotherapy } \\
\hline Absent & 38 & 38 & \multirow{2}{*}{0.170} & 24 & & 25 & \multirow[t]{2}{*}{0.026} \\
\hline Present & 77 & 112 & & 63 & & 62 & \\
\hline Follow up months, median (range) & $37.0(3.2-84.5)$ & $48.4(3.5-89.3)$ & & $37.3(6$ & $-84.5)$ & $\begin{array}{l}46.3 \\
\quad(3.5-89.3)\end{array}$ & \\
\hline
\end{tabular}

$R L N$ Number of retrieved lymph nodes, $S D$ standard deviation, $C E A$ carcinoembryonic antigen, $C A 19-9$ carbohydrate antigen 19-9

${ }^{a}$ Postoperative complications included those of Clavien-Dindo classifications II-V.

to select patients with stage III gastric cancer who underwent total gastrectomy. Stage III gastric cancer is a devastating disease with a poor prognosis, and thus urgently requires the development of better therapeutic strategies and the identification of prognostic factors. Here, when we employed propensity score matching to minimize selection bias before conducting multivariable analysis, we found that the number of RLNs served an independent prognostic factor.

The number of RNLs serves as a prognostic factor for gastric cancer as well as for postoperative survival from certain cancers $[9,15,19,20]$. For example, for colorectal cancer, > 12 RLNs are recommended; and in the absence of $\geq 12$ evaluated nodes, patients should be treated with adjuvant chemotherapy [16]. Moreover, an increase in the number of RLNs is associated with longer survival of patients with node-positive cervical cancer [15]. In 1997, the staging criteria for gastric cancer published by the American Joint Committee on Cancer (AJCC) and the International Union Against Cancer (UICC) were revised to accommodate an $\mathrm{N}$ category assignment, which now reflects the total number of positive LNs, ranging from $\geq 0-15$ [21]. The utility of the prognostic significance of these criteria is validated by several large clinical series [22]. However, it is not established that the optimal number of LNs to be removed and examined achieves optimum reliability in assigning disease stage. Nevertheless, surgeons in countries of the Far East have long recognized the survival benefit of D2 lymphadenectomy, although the concept is not readily accepted in other countries [3, 23]. The Dutch D1D2 trial [1] represents the turning point in establishing the optimal extent of lymphadenectomy for gastric cancer. This trial, which analyzed $>15$ years of results, demonstrates that there are fewer locoregional recurrences of gastric cancer and better long-term survival benefits to patients with D2 lymphadenectomy compared with those with D1 lymphadenectomy. These findings inspired a re-evaluation of the therapeutic value of D2 lymphadenectomy in Western countries [24]. Therefore, the clinical significance of the number of RLNs associated with the extent of lymphadenectomy may be illuminating.

Zhao et al. [18], who analyzed 2246 patients who underwent curative gastrectomy for advanced gastric cancer at a single center, recommend harvesting of $\geq 25$ RLNs. In contrast, the AJCC recommendation ( $\geq 15 \mathrm{RLNs}$ ) is insufficient for determining the $\mathrm{N}$ stage [19]. Zhao et al. [18] argue that more RLNs will be required for total gastrectomy cases [19]. Woo et al. [23] used the Surveillance, Epidemiology, and End Results (SEER) program's database and the Korean database to an analysis of 25,289 patients with gastric cancer [23]. This analysis demonstrates that the maximum survival advantage was achieved by performing a lymphadenectomy with a minimum of 29 RLNs [25]. 


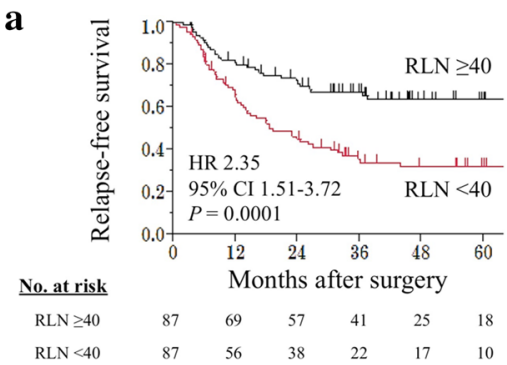

b
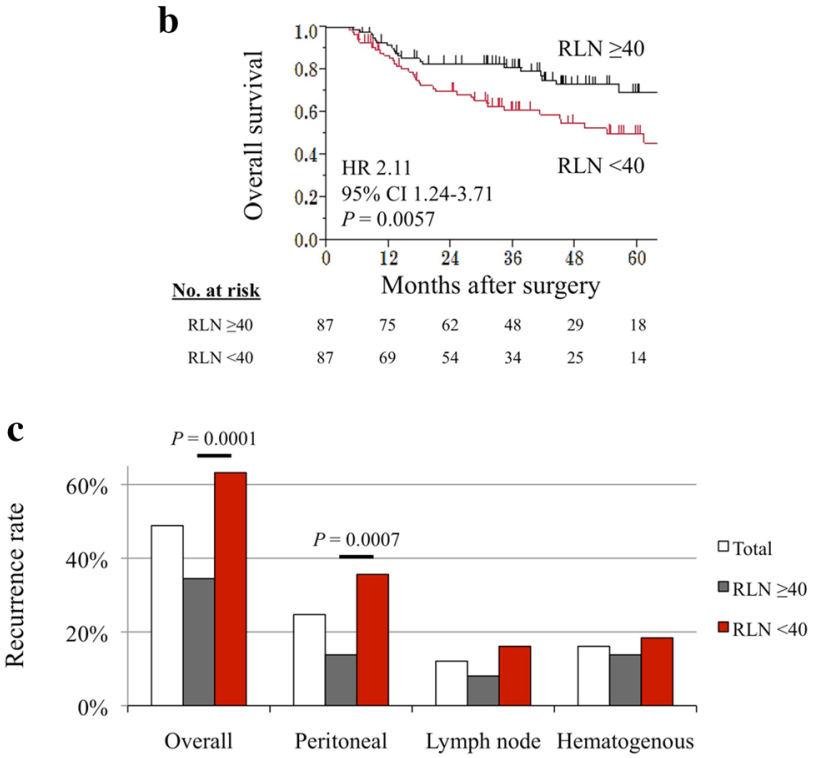

Fig. 2 Prognostic significance of the number of RLNs after propensity score matching. Patients in the RLN $\geq 40$ group were more likely to experience longer relapse-free survival (a) and overall survival (b) compared with those in the RLN $<40$ group. $\mathbf{c}$ Frequencies of sites of initial recurrences

However, these studies are limited by the inconsistent content of the data that include mixed types of gastrectomy, varying extents of lymphadenectomies, and broad disease stages, which likely lead to potential selection bias. Moreover, their consideration of splenectomy and adjuvant treatment is insufficient. In our present study, we minimized selection bias by designing a collaborative database generated from the records of multiple institutions, which included patients who underwent gastrectomy between 2010 and 2014. To avoid potential problems caused by the long accumulation period, we employed propensity score matching. Moreover, the results of ROC analysis led us to focus on patients with stage III gastric cancer.

As we predicted, there were differences in variables such as age and frequency of splenectomy before matching. The patients' backgrounds were well-balanced by the propensity scores calculated for the eight variables listed in the Methods section. Thus, dissection of lymph nodes at the splenic hilum should be comparable between the two groups, because splenectomy was one of the variables used to estimate propensity scores. Moreover, no significant differences in the dissection area (D1 + or D2) were apparent between the two groups after matching.

The number of RNLs is influenced by the extent of lymphadenectomy, the surgeon's enthusiasm to pathologically examine more LNs, the surgeon's skill, surgical considerations (such as fat volume), and the innate number of LNs in each patient $[9-11,18]$. Depending on the surgeon's skill, LNs may not be completely excised, may be destroyed, or both, which may affect the number of RLNs. Further, the number of RLNs reflects the appropriate resection and treatment of specimens. We suggest, therefore, that the $\mathrm{N}$ factor is significantly influenced by a human intervention factor that must represent an important limitation of the presents study as well as a limitation of using the TNM classification system. Another argument states that the number of RLNs reflects the actual number of LNs removed surgically as well as the number of LNs identified and properly examined during macroscopic and microscopic pathological analyses $[15,19,26,27]$. In certain high-volume centers, LNs are retrieved by pathologists who are less familiar with anatomic locations of perigastric LNs $[9,10]$. However, the surgeons who participated in the present study are sufficiently familiar with surgical procedures and the anatomy of RLNs, although individual differences among RLNs exist.

We show here that there were significant associations between the number of RLNs and outcomes of patients with stage III gastric cancer after gastrectomy with systemic lymphadenectomy. Stage migration is inapplicable to the present study because we focused on patients pathologically diagnosed with stage III disease. Further, the possibility of inadequate lymphatic dissection because of the large amount of visceral fat is inapplicable to our study, because sex and body mass indices were adjusted using propensity score matching.

To support our conclusions, we offer several explanations. First, fewer RLNs may simply indicate the existence of unretrieved LNs, and more RLNs may indicate highquality surgery. Second, very small (micro) LNs may have been overlooked and not retrieved from some patients. Distant metastasis (M1 status) may be underestimated if there was metastasis in those micro LNs beyond the area of the D2 lymphadenectomy. Third, RLNs reflect the number of LNs identified and properly examined during macroscopic and microscopic pathologic analyses; therefore, RLNs are a surrogate for particularly functional, favorable practice patterns, which may extend to better follow-up care $[8,18$, 19]. Fourth, there is a possibility that individual differences in the number of LNs are related to prognosis. Fifth, large amounts of perigastric lymphoid tissue may be linked to increased barriers to defense against metastasis and robust tumor immunity $[15,16]$. 
Table 2 Prognostic factors for disease-free survival in patients with stage III gastric cancer who underwent total gastrectomy

\begin{tabular}{|c|c|c|c|c|c|c|}
\hline \multirow[t]{2}{*}{ Variables } & \multicolumn{3}{|c|}{ Univariate } & \multicolumn{3}{|c|}{ Multivariable } \\
\hline & $\mathrm{HR}$ & $95 \% \mathrm{CI}$ & $P$ & HR & $95 \% \mathrm{CI}$ & $P$ \\
\hline \multicolumn{7}{|l|}{ Age } \\
\hline$\geq 65$ & 1.64 & $1.01-2.77$ & 0.0457 & 1.28 & $0.75-2.24$ & 0.3673 \\
\hline \multicolumn{7}{|l|}{ Sex } \\
\hline Male & 1.09 & $0.69-1.77$ & 0.7181 & & & \\
\hline \multicolumn{7}{|c|}{ Preoperative body mass index } \\
\hline$\geq 22$ & 0.80 & $0.51-1.23$ & 0.3062 & & & \\
\hline \multicolumn{7}{|l|}{ Preoperative CEA } \\
\hline$>5 \mathrm{ng} / \mathrm{ml}$ & 1.16 & $0.68-1.90$ & 0.5645 & & & \\
\hline \multicolumn{7}{|l|}{ Preoperative CA19-9 } \\
\hline$>37 \mathrm{IU} / \mathrm{ml}$ & 1.04 & $0.60-1.72$ & 0.8742 & & & \\
\hline \multicolumn{7}{|l|}{ Tumor location } \\
\hline Lower/middle third & 0.89 & $0.57-1.43$ & 0.6300 & & & \\
\hline \multicolumn{7}{|l|}{ Multifocal lesions } \\
\hline Present & 0.73 & $0.18-1.96$ & 0.5785 & & & \\
\hline \multicolumn{7}{|l|}{ Macroscopic type } \\
\hline Borrmann $4 / 5$ & 2.24 & $1.40-3.75$ & 0.0016 & 1.67 & $0.95-2.84$ & 0.0737 \\
\hline \multicolumn{7}{|c|}{ Macroscopic tumor size } \\
\hline$\geq 50 \mathrm{~mm}$ & 1.90 & $1.12-3.46$ & 0.0157 & 1.14 & $0.63-2.16$ & 0.6696 \\
\hline \multicolumn{7}{|l|}{ Splenectomy } \\
\hline Performed & 0.82 & $0.54-1.26$ & 0.3644 & & & \\
\hline \multicolumn{7}{|l|}{ Operative time } \\
\hline$\geq 300 \mathrm{~min}$ & 1.02 & $0.66-1.60$ & 0.9133 & & & \\
\hline \multicolumn{7}{|l|}{ Estimated blood loss } \\
\hline$>400 \mathrm{ml}$ & 1.02 & $0.67-1.57$ & 0.9186 & & & \\
\hline \multicolumn{7}{|c|}{ Intraoperative transfusion } \\
\hline Performed & 1.30 & $0.63-2.41$ & 0.4458 & & & \\
\hline \multicolumn{7}{|c|}{ Number of retrieved lymph nodes } \\
\hline$<40$ & 2.35 & $1.51-3.72$ & 0.0001 & 2.43 & $1.54-3.94$ & 0.0001 \\
\hline \multicolumn{7}{|c|}{ Postoperative complication $^{\mathrm{a}}$} \\
\hline Present & 1.05 & $0.68-1.64$ & 0.8353 & & & \\
\hline \multicolumn{7}{|l|}{ Tumor differentiation } \\
\hline Undifferentiated & 1.21 & $0.78-1.86$ & 0.3830 & & & \\
\hline \multicolumn{7}{|l|}{ Lymphatic involvement } \\
\hline ly $2 / 3$ & 1.34 & $0.81-2.36$ & 0.2650 & & & \\
\hline \multicolumn{7}{|l|}{ Vascular invasion } \\
\hline $\mathrm{v} 2 / 3$ & 1.22 & $0.80-1.88$ & 0.3557 & & & \\
\hline \multicolumn{7}{|l|}{ Infiltrative growth type } \\
\hline Invasive & 1.63 & $1.06-2.53$ & 0.0258 & 1.45 & $0.88-2.38$ & 0.1444 \\
\hline \multicolumn{7}{|c|}{ Pathological tumor depth } \\
\hline pT4 & 1.27 & $0.81-2.06$ & 0.3023 & & & \\
\hline Pathological lymph n & netast: & & & & & \\
\hline $\mathrm{pN} 3$ & 2.85 & $1.84-4.46$ & $<0.0001$ & 2.72 & $1.67-4.50$ & $<0.0001$ \\
\hline Adjuvant chemothera & & & & & & \\
\hline Performed & 0.53 & $0.34-0.86$ & 0.0114 & 0.50 & $0.30-0.86$ & 0.0135 \\
\hline
\end{tabular}

HR Hazard ratio, CI confidence interval, CEA carcinoembryonic antigen, CA19-9 carbohydrate antigen 19-9

${ }^{\mathrm{a}}$ Grade II-V in the Clavien-Dindo classification 


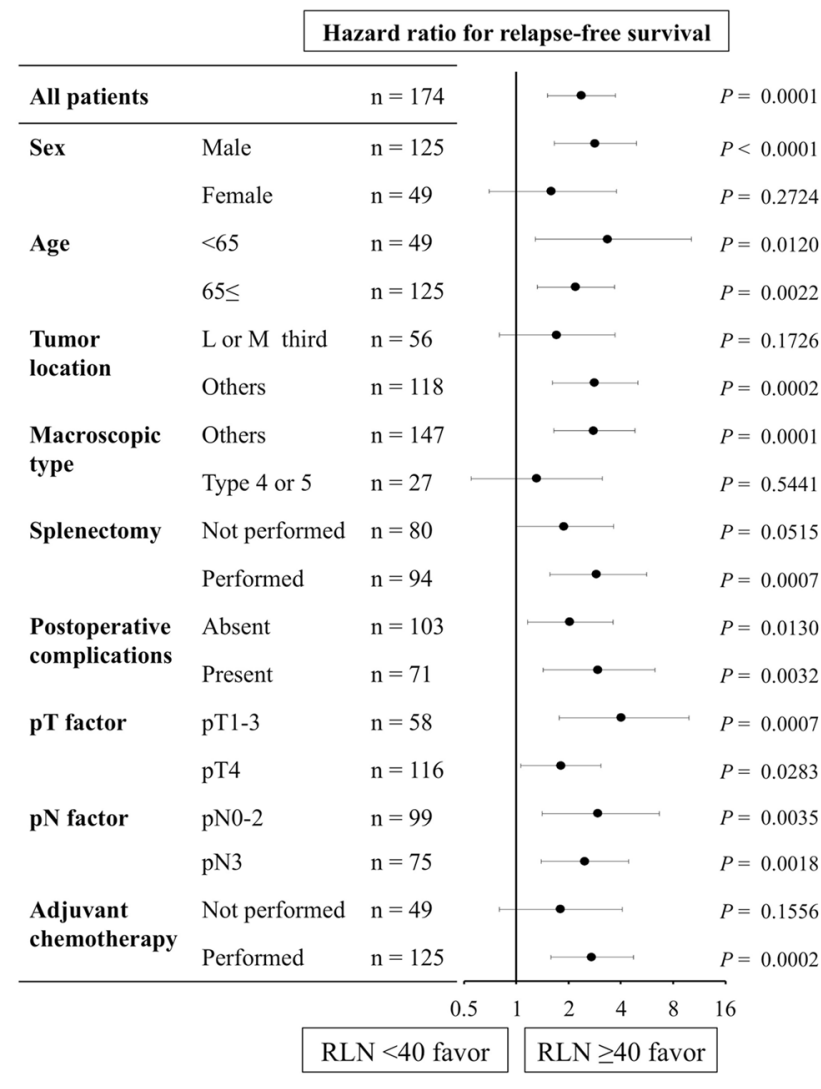

Fig. 3 Significance of the association of the number of RLNs with relapse-free survival

We believe that our findings of a relative and significant increase in the prevalence of nodal occurrence in the $\mathrm{RLN}<40$ group are not surprising. However, we were interested to find that the prevalence of peritoneal recurrence was significantly greater in the RLN $<40$ group. One explanation is that a smaller number of RLNs reflects the destruction of LNs during surgery, causing the dissemination of cancer cells into the peritoneal cavity. Moreover, if the number of RLNs reflects a surgeon's skill, inappropriate treatment of serosa-invading gastric cancer during surgery may disseminate cancer cells from the primary tumors.

Convincing evidence indicates that adjuvant chemotherapy contributes to prolonged survival after surgery for stage III gastric cancer $[4,28]$. In the present study, we found that the 5-year relapse-free survival rate was $>70 \%$, even in patients with stage III gastric cancer, if the number of RLNs was $\geq 40$ and adjuvant chemotherapy was administered. In contrast, the RLN $<40$ group had a poor prognosis regardless of postoperative adjuvant chemotherapy, indicating that a small number of RLNs was a disadvantage because it was not compensated by adjuvant treatment. Splenectomy typically achieves increased numbers of RLNs. However, our results do not indicate that splenectomy compensated for the adverse effect of fewer RLNs on prognosis. $\mathbf{a}$

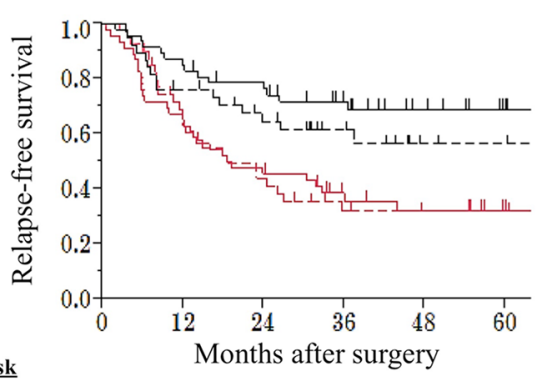

\begin{tabular}{ccccccc} 
No. at risk & & & & & \\
\hline - RLN $\geq 40$, splenectomy + & 48 & 41 & 36 & 27 & 18 & 12 \\
- - - . RLN $\geq 40$, splenectomy - & 39 & 29 & 22 & 15 & 8 & 7 \\
\hline - - RLN $<40$, splenectomy + & 46 & 32 & 22 & 14 & 11 & 5 \\
RLN $<40$, splenectomy - & 41 & 25 & 18 & 9 & 7 & 6
\end{tabular}

b

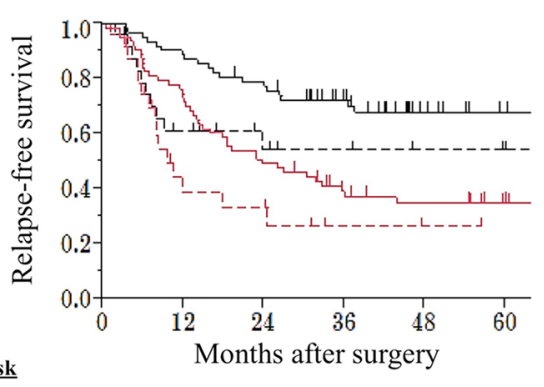

\begin{tabular}{|c|c|c|c|c|c|c|c|}
\hline & No. at risk & & & 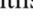 & $x_{1}$ & 3 & \\
\hline & $R L N \geq 40$, adjuvant & 62 & 56 & 49 & 35 & 21 & 15 \\
\hline--- & $R L N \geq 40$, surgery alone & 25 & 14 & 9 & 7 & 5 & 4 \\
\hline & RLN $<40$, adjuvant & 63 & 48 & 32 & 20 & 16 & 10 \\
\hline--- & RLN $<40$, surgery alone & 24 & 8 & 7 & 3 & 2 & 0 \\
\hline
\end{tabular}

Fig. 4 Analysis of relapse-free survival curves of patient groups categorized according to RLNs and splenectomy (a) or administration of postoperative adjuvant chemotherapy (b)

To translate our findings to clinical practice, our data should encourage surgeons to perform appropriate lymphadenectomies, aiming to achieve RLNs $\geq 40$ without destruction of LNs. Moreover, the number of RLNs is a useful predictive marker for risk stratification [18-20]. For patients with RLNs $<40$, careful and intensive follow-up should be performed, because they are at high risk of disease recurrence. The UICC guidelines recommend radiotherapy and chemotherapy to prevent recurrence in patients with $>16$ harvested LNs [11]. Postoperative adjuvant chemoradiation therapy may, therefore, serve as a preferred option to control locoregional recurrence and suitable for evaluating patients with RLNs $<40$ after total gastrectomy, though this is not been proven to confer a survival benefit upon patients from Asian countries [28-30].

Our study has several limitations. First, this was a retrospective study, and the AUC value (0.6035) was relatively low to achieve the optimum initial selection of the target population. Second, we analyzed data only for Japanese patients. Third, the quality of the RLNs harvested at each institution may have differed, because the surgical procedure 
was not completely described in patients' records. Fourth, little information was available on LN dissection at the splenic hilum without splenectomy. Fifth, because S-1 monotherapy was administered to most patients, it was difficult to evaluate the correlations between the number of RLNs and doublet chemotherapy (e.g. capecitabine plus oxaliplatin).

\section{Conclusion}

In summary, our results indicate that the number of RLNs served as an independent prognostic factor after total gastrectomy administered to patients with stage III gastric cancer. Efforts should, therefore, be made to acquire $\geq 40$ RLNs, and intensive disease management is advisable for patients with RLNs $<40$.

Acknowledgements We thank Edanz Group (http://www.edanzediti ng.com/ac) for editing a draft of this manuscript.

\section{Compliance with ethical standards}

Ethical standards All procedures followed were in accordance with the ethical standards of the responsible committee on human experimentation (institutional and national) and with the Helsinki Declaration of 1964 and later versions. Informed consent to be included in the study, or the equivalent, was obtained from all patients.

Conflict of interest All Authors declare that they have no conflict of interest.

\section{References}

1. Leung WK, Wu MS, Kakugawa Y, Kim JJ, Yeoh KG, Goh KL, et al. Screening for gastric cancer in Asia: current evidence and practice. Lancet Oncol. 2008;9:279-87.

2. Gonzalez CA, Agudo A. Carcinogenesis, prevention and early detection of gastric cancer: where we are and where we should go. Int J Cancer. 2012;130:745-53.

3. Van Cutsem E, Sagaert X, Topal B, Haustermans K, Prenen H. Gastric cancer. Lancet. 2016;388:2654-64.

4. Foo M, Leong T. Adjuvant therapy for gastric cancer: current and future directions. World J Gastroenterol. 2014;20:13718-27.

5. Kanda M, Kodera Y, Sakamoto J. Updated evidence on adjuvant treatments for gastric cancer. Expert Rev Gastroenterol Hepatol. 2015;9:1-12

6. Sasako M, Sano T, Yamamoto S, Kurokawa Y, Nashimoto A, Kurita A, et al. D2 lymphadenectomy alone or with paraaortic nodal dissection for gastric cancer. N Engl J Med. 2008;359:453-62.

7. Japanese Gastric Cancer Association. Japanese gastric cancer treatment guidelines 2014 (ver. 4). Gastric Cancer. 2017;20:1-19.

8. Eom BW, Joo J, Kim YW, Reim D, Park JY, Yoon HM, et al. Improved survival after adding dissection of the superior mesenteric vein lymph node $(14 \mathrm{v})$ to standard D2 gastrectomy for advanced distal gastric cancer. Surgery. 2014;155:408-16.
9. Li F, Hong X, Hou L, Lin F, Chen P, Pang S, et al. A greater number of dissected lymph nodes is associated with more favorable outcomes in bladder cancer treated by radical cystectomy: a meta-analysis. Oncotarget. 2016;7:61284-94.

10. Smith DD, Schwarz RR, Schwarz RE. Impact of total lymph node count on staging and survival after gastrectomy for gastric cancer: data from a large US-population database. J Clin Oncol. 2005;23:7114-24.

11. Jiang L, Yao Z, Zhang Y, Hu J, Zhao D, Zhai H, et al. Comparison of lymph node number and prognosis in gastric cancer patients with perigastric lymph nodes retrieved by surgeons and pathologists. Chin J Cancer Res. 2016;28:511-8.

12. Liu JY, Peng CW, Yang XJ, Huang CQ, Li Y. The prognosis role of AJCC/UICC 8(th) edition staging system in gastric cancer, a retrospective analysis. Am J Transl Res. 2018;10:292-303.

13. Kanda M, Shimizu D, Tanaka H, Tanaka C, Kobayashi D, Hayashi M, et al. Significance of SYT8 for the detection, prediction, and treatment of peritoneal metastasis from gastric cancer. Ann Surg. 2018;267:495-503.

14. Miki Y, Tokunaga M, Tanizawa Y, Bando E, Kawamura T, Terashima M. Perioperative risk assessment for gastrectomy by surgical apgar score. Ann Surg Oncol. 2014;21:2601-7.

15. Zhou J, Zhang WW, Wu SG, He ZY, Sun JY, Wang Y, et al. The impact of examined lymph node count on survival in squamous cell carcinoma and adenocarcinoma of the uterine cervix. Cancer Manag Res. 2017;9:315-22.

16. Degiuli M, Arolfo S, Evangelista A, Lorenzon L, Reddavid R, Staudacher C, et al. Number of lymph nodes assessed has no prognostic impact in node-negative rectal cancers after neoadjuvant therapy. Results of the "Italian Society of Surgical Oncology (S.I.C.O.) Colorectal Cancer Network" (SICO-CCN) multicentre collaborative study. Eur J Surg Oncol. 2018;44:1233-40.

17. Kanda M, Murotani K, Kobayashi D, Tanaka C, Yamada S, Fujii $\mathrm{T}$, et al. Postoperative adjuvant chemotherapy with $\mathrm{S}-1$ alters recurrence patterns and prognostic factors among patients with stage II/III gastric cancer: a propensity score matching analysis. Surgery. 2015;158:1573-80.

18. Okajima W, Komatsu S, Ichikawa D, Kosuga T, Kubota T, Okamoto K, et al. Prognostic impact of the number of retrieved lymph nodes in patients with gastric cancer. J Gastroenterol Hepatol. 2016;31:1566-71

19. Zhao B, Zhang J, Chen X, Sun T, Wang Z, Xu H, et al. The retrieval of at least 25 lymph nodes should be essential for advanced gastric cancer patients with lymph node metastasis: a retrospective analysis of single-institution database study design: Cohort study. Int J Surg. 2017;48:291-9.

20. Hall MD, Schultheiss TE, Smith DD, Fakih MG, Kim J, Wong JY, et al. Impact of total lymph node count on staging and survival after neoadjuvant chemoradiation therapy for rectal cancer. Ann Surg Oncol. 2015;22(Suppl 3):580-7.

21. Wu CW, Hsieh MC, Lo SS, Shen KH, Lui WY, P'Eng FK. Comparison of the UICC/AJCC 1992 and $1997 \mathrm{pN}$ categories for gastric cancer patients after surgery. Hepatogastroenterology. 2001;48:279-84.

22. Karpeh MS, Leon L, Klimstra D, Brennan MF. Lymph node staging in gastric cancer: is location more important than Number? An analysis of 1038 patients. Ann Surg. 2000;232:362-71.

23. Hartgrink HH, van de Velde CJ, Putter H, Bonenkamp JJ, Klein Kranenbarg E, Songun I, et al. Extended lymph node dissection for gastric cancer: who may benefit? Final results of the randomized Dutch gastric cancer group trial. J Clin Oncol. 2004:22:2069-77.

24. Songun I, Putter H, Kranenbarg EM, Sasako M, van de Velde CJ. Surgical treatment of gastric cancer: 15-year follow-up results of the randomised nationwide Dutch D1D2 trial. Lancet Oncol. 2010;11:439-49. 
25. Woo Y, Goldner B, Ituarte P, Lee B, Melstrom L, Son T, et al. Lymphadenectomy with optimum of 29 lymph nodes retrieved associated with improved survival in advanced gastric cancer: a 25,000-Patient International Database Study. J Am Coll Surg. 2017;224:546-55.

26. Chen T, Yan D, Zheng Z, Yang J, Dong XDE. Evolution in the surgical management of gastric cancer: is extended lymph node dissection back in vogue in the USA? World J Surg Oncol. 2017; $15: 135$

27. Lu J, Wang W, Zheng CH, Fang C, Li P, Xie JW, et al. Influence of total lymph node count on staging and survival after gastrectomy for gastric cancer: an analysis from a two-institution database in China. Ann Surg Oncol. 2017;24:486-93.
28. Ashraf N, Hoffe S, Kim R. Adjuvant treatment for gastric cancer: chemotherapy versus radiation. Oncologist. 2013;18:1013-21.

29. Macdonald JS, Smalley SR, Benedetti J, Hundahl SA, Estes NC, Stemmermann GN, et al. Chemoradiotherapy after surgery compared with surgery alone for adenocarcinoma of the stomach or gastroesophageal junction. N Engl J Med. 2001;345:725-30.

30. Wong RK, Jang R, Darling G. Postoperative chemoradiotherapy vs. preoperative chemoradiotherapy for locally advanced (operable) gastric cancer: clarifying the role and technique of radiotherapy. J Gastrointest Oncol. 2015;6:89-107. 